\title{
On a Nonlocal Ostrovsky-Whitham Type Dynamical System, Its Riemann Type Inhomogeneous Regularizations and Their Integrability
}

\author{
Jotanta GOLENIA ${ }^{\dagger^{1}}$, Maxim V.PAVLOV ${ }^{\dagger^{2}}$, \\ Ziemowit POPOWICZ $\dagger^{\dagger^{3}}$ and Anatoliy K. PRYKARPATSKY $Y^{\dagger^{4} \dagger^{5}}$ \\ $\dagger^{1}$ The Department of Applied Mathematics, AGH University of Science and Technology, \\ Kraków 30059, Poland \\ E-mail: golenia@agh.edu.pl \\ $\dagger^{2}$ Department of Mathematical Physics, P.N. Lebedev Physical Institute, \\ 53 Leninskij Prospekt, Moscow 119991, Russia \\ E-mail: M.V.Pavlov@lboro.ac.uk \\ $\dagger^{3}$ The Institute for Theoretical Physics, University of Wrockaw, Wrockaw 50204, Poland \\ E-mail: ziemek@ift.uni.wroc.pl \\ URL: www.ift.uni.wroc.pl/ ziemek/ \\ $\dagger^{4}$ The Department of Mining Geodesics, AGH University of Science and Technology, \\ Kraków 30059, Poland \\ $\dagger^{5}$ Department of Economical Cybernetics, Ivan Franko State Pedagogical University, \\ Drohobych, Lviv Region, Ukraine \\ E-mail:pryk.anat@ua.fm
}

Received October 14, 2009, in final form January 03, 2010; Published online January 07, 2010 doi:10.3842/SIGMA.2010.002

\begin{abstract}
Short-wave perturbations in a relaxing medium, governed by a special reduction of the Ostrovsky evolution equation, and later derived by Whitham, are studied using the gradient-holonomic integrability algorithm. The bi-Hamiltonicity and complete integrability of the corresponding dynamical system is stated and an infinite hierarchy of commuting to each other conservation laws of dispersive type are found. The well defined regularization of the model is constructed and its Lax type integrability is discussed. A generalized hydrodynamical Riemann type system is considered, infinite hierarchies of conservation laws, related compatible Poisson structures and a Lax type representation for the special case $N=3$ are constructed.
\end{abstract}

Key words: generalized Riemann type hydrodynamical equations; Whitham type dynamical systems; Hamiltonian systems; Lax type integrability; gradient-holonomic algorithm

2010 Mathematics Subject Classification: 35C05; 37K10

\section{Introduction}

Many important problems of propagating waves in nonlinear media with distributed parameters, for instance, invisible non-dissipative dark matter, playing a decisive role $[9,10]$ in the formation of large scale structure in the Universe like galaxies, clusters of galaxies, super-clusters, can be described by means of evolution differential equations of special type. It is also well known [2,

*This paper is a contribution to the Proceedings of the Eighth International Conference "Symmetry in Nonlinear Mathematical Physics" (June 21-27, 2009, Kyiv, Ukraine). The full collection is available at http://www.emis.de/journals/SIGMA/symmetry2009.html 
$17,25,28]$ that shortwave perturbations in a relaxing one dimensional medium can be described by means of some reduction of the Ostrovsky equations, coinciding with the Whitham type evolution equation

$$
d u / d t=2 u u_{x}+\int_{\mathbb{R}} \mathcal{K}(x, s) u_{s} d s,
$$

discussed first in [28]. Here the kernel $\mathcal{K}: \mathbb{R} \times \mathbb{R} \rightarrow \mathbb{R}$ depends on the medium elasticity properties with spatial memory and can, in general, be a function of the pressure gradient $u_{x} \in C^{\infty}(\mathbb{R} ; \mathbb{R})$, evolving with respect to equation (1.1). In particular, if the nonlinear medium is endowed still with spatial memory properties, that is the wave amplitude depends on the orbit, swept by its front, the propagation of the corresponding wave can be modeled by means of the so called generalized Ostrovsky evolution equations [20]. Namely, if to put $\mathcal{K}(x, s)=\frac{1}{2}|x-s|, x, s \in \mathbb{R}$, then equation (1.1) can be reduced to

$$
d u / d t=2 u u_{x}+\partial^{-1} u,
$$

which was, in particular, studied before in [17, 21, 22].

Since some media possess elasticity properties depending strongly on the spatial pressure gradient $u_{x}, x \in \mathbb{R}$, the corresponding Whitham type kernel looks like

$$
\mathcal{K}(x, s):=-\theta(x-s) u_{s}
$$

for $x, s \in \mathbb{R}$, naturally modeling the relaxing spatial memory effects. The resulting equation (1.1) with the kernel (1.2) becomes

$$
d u / d t=2 u u_{x}-\partial^{-1} u_{x}^{2}:=K[u],
$$

which appears to possess very interesting mathematical properties. The latter will be the main topic of the next sections below.

Owing to the results, obtained before in [25, 26], the dynamical system (1.3) appeared to be a Lax type integrable bi-Hamiltonian flow, but with ill posed temporal evolution. As it was demonstrated in [26], a suitable finite-dimensional reduction scheme, if applied to the corresponding hierarchy of conservation laws for constructing explicit solutions to the Ostrovsky-Whitham type nonlinear dynamical system (1.3) by means of quadratures, meets some technical problems. Some of these integrability aspects were before presented in [2], where a suitable well posed regularization of the equation (1.3) in the form

$$
\left.\begin{array}{l}
u_{t}=2 u u_{x}-v \\
v_{t}=2 u v_{x}
\end{array}\right\}:=K[u, v]
$$

for treating this nonlocality problem was proposed.

Below the well posed integrability problem for the Ostrovsky-Whitham type nonlinear and nonlocal dynamical system (1.4) will be reanalyzed in detail making use of this regularization scheme. The corresponding implectic structures and Lax type representations are found by means of the differential-geometric tools, devised and extended in [7, 8, 15, 24]. A natural Riemann type generalization of the dynamical system (1.4) is proposed, owing to a recent observation by D. Holm and M. Pavlov:

$$
D_{t}^{N} u=0, \quad N \in \mathbb{Z}_{+},
$$

which at $N=2$ is exactly equivalent to the system (1.4). The integrability properties of equation (1.5) at $N=3$ were analyzed in detail, the conservation laws, corresponding compatible implectic structures and Lax type representation are constructed. 
It is worth to mention that the obtained in this work Lax type pair (3.6) for the regularized dynamical system (1.4) was found first in work [4]. It coincides with those found later in [23], making use of a very special bi-Lagrangian representation of the dynamical system (1.4). But the existence of the singular co-implectic structure (3.14) in these references was not stated. A detailed analysis of the relationships between solutions of dynamical systems (1.3) and (1.4), based on a reciprocal transformation, suggested by M. Pavlov in [23], was presented recently in [27]. Mention also work [14], where the geometric aspects of the equation like (2.1) were studied.

Note also here that theory of integrable homogenous hydrodynamic type systems with distinct characteristic velocities was constructed by S.P. Tsarev. In this paper we consider the first example in a literature of nonhomogeneous integrable hydrodynamic type systems with a sole characteristic velocity. Such a theory does not exist at this moment.

\section{A regularization scheme and the geometric integrability problem}

Define a smooth periodic function $v \in C_{2 \pi}^{\infty}(\mathbb{R} ; \mathbb{R})$, such that

$$
v:=\partial^{-1} u_{x}^{2}
$$

for any $x, t \in \mathbb{R}$, where the function $u \in C_{2 \pi}^{\infty}(\mathbb{R} ; \mathbb{R})$ solves equation (1.3). Then it is easy to state that the following regularized nonlinear dynamical system

$$
\left.\begin{array}{l}
u_{t}=2 u u_{x}-v \\
v_{t}=2 u v_{x}
\end{array}\right\}:=K[u, v]
$$

of hydrodynamic type, which was introduced before in [6], studied in [2, 4, 12, 13, 19] and analyzed as a Gurevich-Zybin system in [23], and is already well defined on the extended $2 \pi$-periodic functional space $\mathcal{M}:=C_{2 \pi}^{\infty}\left(\mathbb{R} ; \mathbb{R}^{2}\right)$ and equivalent on the functional submanifold $\mathcal{M}_{\text {red }}:=\left\{(u, v) \in \mathcal{M}: v_{x}-u_{x}^{2}=0\right\}$ to that given by expression (1.3), as it was mentioned in [2] and discussed recently in [27]. The system (2.1) can be rewritten as the following set of equations

$$
\begin{aligned}
& u_{t}=2 u u_{x}-v, \quad v_{t}=2 u v_{x}, \\
& u_{x}=w, v_{x}=u_{x} w \\
& w_{t}=v_{x}+2 u w_{x}
\end{aligned}
$$

which is equivalent to a set of differential two-forms

$$
\begin{aligned}
\{\alpha\}:=\left\{\begin{array}{c}
\alpha^{(1)} \\
=
\end{array}\right) d u \wedge d x+2 u d u \wedge d t-v d x \wedge d t, \alpha^{(2)}=d v \wedge d x+2 u d v \wedge d t, \\
\alpha^{(3)}=d u \wedge d t-w d x \wedge d t, \alpha^{(4)}=d v \wedge d t-w d u \wedge d t, \\
\left.\alpha^{(5)}=d w \wedge d x+d v \wedge d t+2 u d w \wedge d t\right\} .
\end{aligned}
$$

This set of two-forms generates the closed ideal $\mathcal{I}(\alpha)$, since

$$
\begin{aligned}
& d \alpha^{(1)}=-\alpha^{(2)} \wedge d t, \quad d \alpha^{(2)}=2 d u \wedge \alpha^{(4)}, \quad d \alpha^{(3)}=-\alpha^{(5)} \wedge d t, \\
& d \alpha^{(4)}=-d w \wedge \alpha^{(3)}-w d t \wedge \alpha^{(5)}, \quad d \alpha^{(5)}=-2 d w \wedge \alpha^{(3)}-2 w d t \wedge \alpha^{(5)} .
\end{aligned}
$$

The set of differential forms (2.3), being integrable, defines the integral submanifold $\bar{M}$ by means of the condition $\mathcal{I}(\alpha)=0$. Making now use of the differential-geometric method devised 
in $[11,18,24]$ and extending algorithmically the approach of [15], we will look for a reduced upon the integral submanifold $\bar{M}$ connection one-form $\Gamma$, belonging to some not yet determined its holonomy Lie algebra $\mathcal{G}$. This 1 -form can be represented as follows:

$$
\Gamma=\mathcal{A}(u, v, w) d x+\mathcal{B}(u, v, w) d t
$$

where the elements $\mathcal{A}, \mathcal{B} \in \mathcal{G}$ satisfy determining equations

$$
\begin{aligned}
\Omega= & \frac{\partial \mathcal{A}}{\partial u} d u \wedge d x+\frac{\partial \mathcal{A}}{\partial v} d v \wedge d x+\frac{\partial \mathcal{A}}{\partial w} d w \wedge d x+\frac{\partial \mathcal{B}}{\partial u} d u \wedge d t \\
& +\frac{\partial \mathcal{B}}{\partial v} d v \wedge d t+\frac{\partial \mathcal{B}}{\partial w} d w \wedge d t+[\mathcal{A}, \mathcal{B}] d x \wedge d t \\
\Rightarrow \quad & g_{1}(d u \wedge d x+2 u d u \wedge d t-v d x \wedge d t)+g_{2}(d v \wedge d x+2 u d v \wedge d t) \\
& +g_{3}(d u \wedge d t-w d x \wedge d t)+g_{4}(d v \wedge d t-w d u \wedge d t) \\
& +g_{5}(d w \wedge d x+2 u d w \wedge d t+d v \wedge d t) \in \mathcal{I}(\alpha) \otimes \mathcal{G}
\end{aligned}
$$

for some $\mathcal{G}$-valued functions $g_{1}, \ldots, g_{5} \in \mathcal{G}$ on $M$. From (2.5) one finds that

$$
\begin{aligned}
& \frac{\partial \mathcal{A}}{\partial u}=g_{1}, \quad \frac{\partial \mathcal{A}}{\partial v}=g_{2}, \quad \frac{\partial \mathcal{A}}{\partial w}=g_{5}, \\
& \frac{\partial \mathcal{B}}{\partial u}=2 u g_{1}+g_{3}-w g_{4}, \quad \frac{\partial \mathcal{B}}{\partial v}=2 u g_{2}+g_{4}+g_{5}, \\
& \frac{\partial \mathcal{B}}{\partial w}=2 u g_{5}, \quad[\mathcal{A}, \mathcal{B}]=-v g_{1}-w g_{3} .
\end{aligned}
$$

Thereby, from the obtained set of relationships (2.6) one can find that

$$
\begin{aligned}
& \mathcal{B}=2 u \mathcal{A}+\mathcal{C}(u, v), \quad g_{4}=\frac{\partial \mathcal{C}}{\partial v}-\frac{\partial \mathcal{A}}{\partial w}, \quad g_{3}=2 \mathcal{A}+\frac{\partial \mathcal{C}}{\partial u}+w \frac{\partial \mathcal{C}}{\partial v}-w \frac{\partial \mathcal{A}}{\partial w}, \\
& {[\mathcal{A}, \mathcal{C}]=-v \frac{\partial \mathcal{A}}{\partial u}-2 w \mathcal{A}-w \frac{\partial \mathcal{C}}{\partial u}-w^{2} \frac{\partial \mathcal{C}}{\partial v}+w^{2} \frac{\partial \mathcal{A}}{\partial w}}
\end{aligned}
$$

serving for final searching for connection (2.4).

\section{The bi-Hamiltonian structure and Lax-type representation}

Consider the following polynomial expansion of the element $\mathcal{A}(u, v ; w) \in \mathcal{G}$ with respect to the variable $w$ :

$$
\mathcal{A}=\mathcal{A}_{0}(u, v)+\mathcal{A}_{1}(u, v) w+\mathcal{A}_{2}(u, v) w^{2}
$$

and substitute it into the last equation of (2.6). As a result we obtain:

$$
\begin{aligned}
& {\left[\mathcal{A}_{0}, C\right]=-v \frac{\partial \mathcal{A}_{0}}{\partial u}, \quad\left[\mathcal{A}_{1}, C\right]=-v \frac{\partial \mathcal{A}_{1}}{\partial u}-2 \mathcal{A}_{0}-\frac{\partial C}{\partial u},} \\
& {\left[\mathcal{A}_{2}, C\right]=-v \frac{\partial \mathcal{A}_{2}}{\partial u}-\frac{\partial C}{\partial v}-\mathcal{A}_{1}}
\end{aligned}
$$

or

$$
\mathcal{A}_{1}=\left[C, \mathcal{A}_{2}\right]-v \frac{\partial \mathcal{A}_{2}}{\partial u}-\frac{\partial C}{\partial v} .
$$

which can be substituted into the second equation of (3.1):

$$
\left[\left[C, \mathcal{A}_{2}\right], C\right]-2 v\left[\frac{\partial \mathcal{A}_{2}}{\partial u}, C\right]-\left[\frac{\partial C}{\partial v}, C\right]=-v\left[\frac{\partial C}{\partial u}, \mathcal{A}_{2}\right]-v^{2} \frac{\partial^{2} \mathcal{A}_{2}}{\partial u^{2}}-v \frac{\partial^{2} C}{\partial u \partial v}-2 \mathcal{A}_{0}-\frac{\partial C}{\partial u} .
$$


Thus, recalling (3.1) and (3.2), we have that

$$
\begin{aligned}
& 2 \mathcal{A}_{0}=\left[C,\left[C, \mathcal{A}_{2}\right]\right]+2 v\left[\frac{\partial \mathcal{A}_{2}}{\partial u}, C\right]+\left[\frac{\partial C}{\partial v}, C\right]-v\left[\frac{\partial C}{\partial u}, \mathcal{A}_{2}\right]-v^{2} \frac{\partial^{2} \mathcal{A}_{2}}{\partial u^{2}}-v \frac{\partial^{2} C}{\partial u \partial v}-\frac{\partial C}{\partial u}, \\
& {\left[\mathcal{A}_{0}, C\right]=-v \frac{\partial \mathcal{A}_{0}}{\partial u}, \quad \mathcal{A}_{1}=\left[C, \mathcal{A}_{2}\right]-v \frac{\partial \mathcal{A}_{2}}{\partial u}-\frac{\partial C}{\partial v} .}
\end{aligned}
$$

Now we will assume that the element $C:=C_{0}$ is constant and the elements $\mathcal{A}_{0}$ and $\mathcal{A}_{2}$ are linear with respect to variables $u$ and $v$, that is

$$
\mathcal{A}_{0}=\mathcal{A}_{0}^{(0)}+\mathcal{A}_{0}^{(1)} u+\mathcal{A}_{0}^{(2)} v, \quad \mathcal{A}_{2}=\mathcal{A}_{2}^{(0)}+\mathcal{A}_{2}^{(1)} u+\mathcal{A}_{2}^{(2)} v .
$$

Whence and from (3.3) one gets:

$$
\begin{array}{ll}
2 \mathcal{A}_{0}^{(0)}=\left[C_{0},\left[C_{0}, \mathcal{A}_{2}^{(0)}\right]\right], & {\left[\mathcal{A}_{0}^{(1)}, C_{0}\right]=0, \quad\left[\mathcal{A}_{0}^{(2)}, C_{0}\right]=-\mathcal{A}_{0}^{(1)},} \\
2 \mathcal{A}_{0}^{(1)}=\left[C_{0},\left[C_{0}, \mathcal{A}_{2}^{(1)}\right]\right], & 2 \mathcal{A}_{0}^{(2)}=\left[C_{0},\left[C_{0}, \mathcal{A}_{2}^{(2)}\right]\right]+2\left[\mathcal{A}_{2}^{(1)}, C_{0}\right] .
\end{array}
$$

To solve the algebraic system (3.4) we need to calculate [24] the corresponding holonomy Lie algebra of the connection (2.4). As a result of simple, but slightly cumbersome calculations, we derive that elements $\mathcal{A}_{2}^{(j)}, j=0, \ldots, 2$, and $C_{0}$ belong to the Lie algebra $\operatorname{sl}(2 ; \mathbb{C})$, whose basis $L_{0}, L_{+}$and $L_{-}$can be taken to satisfy the following canonical commutation relations:

$$
\left[L_{0}, L_{ \pm}\right]= \pm L_{ \pm}, \quad\left[L_{+}, L_{-}\right]=2 L_{0}
$$

Thereby, making use of the standard determining expansions

$$
\mathcal{A}_{2}^{(j)}=\sum_{ \pm} c_{ \pm}^{(j)} L_{ \pm}+c_{0}^{(j)} L_{0}, \quad C_{0}=\sum_{ \pm} k_{ \pm} L_{ \pm}+k_{0} L_{0}
$$

where $j=0, \ldots, 2$, and substituting (3.5) into (3.4), we obtain some relationships on values $c_{ \pm}^{(j)}, c_{0}^{(j)} \in \mathbb{C}, j=0, \ldots, 2$, and $k_{ \pm}, k_{0} \in \mathbb{C}$. Resolving by means of simple but slightly cumbersome calculations these relationships, we find the searched for basic elements $\mathcal{A}$ and $\mathcal{B}$ of the connection $\Gamma$, depending on a spectral parameter $\lambda \in \mathbb{C}$, thereby giving rise to the corresponding Lax type commutative spectral representation for dynamical system (2.1) in the following $(2 \times 2)$-matrix form:

$$
\begin{aligned}
& \frac{d f}{d x}=\ell[u, v ; \lambda] f, \quad \frac{d f}{d t}=p(\ell) f, \quad p(\ell):=2 u \ell[u, v ; \lambda]+q, \\
& \ell[u, v ; \lambda]:=\left(\begin{array}{cc}
-\lambda u_{x} & -v_{x} \\
\lambda^{2} & \lambda u_{x}
\end{array}\right), \quad q:=\left(\begin{array}{cc}
0 & 0 \\
\lambda & 0
\end{array}\right), \quad p(\ell)=\left(\begin{array}{cc}
-2 \lambda u_{x} u & -2 v_{x} u \\
\lambda+2 \lambda^{2} u & 2 \lambda u_{x} u
\end{array}\right),
\end{aligned}
$$

defining the generalized time-independent spectrum $\operatorname{Spec}(\ell) \subset \mathbb{C}: \lambda \in \operatorname{Spec}(\ell)$, if the corresponding solution $f \in L_{\infty}\left(\mathbb{R} ; \mathbb{C}^{2}\right)$. It is worth to remark here that the Lax type representation (3.6), found for the dynamical system (2.1), is not unique. Moreover, making use of other imbeddings of the connection form (2.4) into a suitable holonomy Lie algebra $\mathcal{G}$, one can construct different Lax type representations, which could appear to be more useful for finding exact solutions to dynamical system (2.1) by means of, for instance, the inverse spectral transform method.

The standard Riccati equation, derived from (3.6), allows to obtain right away an infinite hierarchy of local conservation laws:

$$
\hat{\gamma}_{-1}:=\int_{0}^{2 \pi} \sqrt{u_{x}^{2}-v_{x}} d x, \quad \hat{\gamma}_{0}:=\int_{0}^{2 \pi} \frac{\left(u_{x} v_{x x}-v_{x} u_{x x}\right)}{2 v_{x} \sqrt{u_{x}^{2}-v_{x}}} d x, \quad \ldots
$$


and so on. All of conservation laws (3.7) except $\gamma_{-1}$, are singular at the Cauchy condition (2.2). This means that we need to construct other hierarchy of polynomial conservation laws regular on the functional submanifold

$$
\mathcal{M}_{\text {red }}:=\left\{(u, v) \in \mathcal{M}: u_{x}^{2}-v_{x}=0, x \in \mathbb{R} / 2 \pi \mathbb{Z}\right\} .
$$

The latter exists owing to the results of $[23,24]$. The simplest way to search for them consists in determining the bi-Hamiltonian structure of flow (2.1). As it is easy to check, dynamical system (2.1) is canonically Hamiltonian, that is

$$
\frac{d}{d t}(u, v)^{\top}:=-\hat{\vartheta} \operatorname{grad} \hat{H}_{\vartheta}=\hat{K}[u, v]
$$

where the corresponding co-symplectic structure $\hat{\vartheta}: T^{*}(\mathcal{M}) \rightarrow T(\mathcal{M})$ is canonical, equals

$$
\hat{\vartheta}=\left(\begin{array}{cc}
0 & 1 \\
-1 & 0
\end{array}\right)
$$

and satisfies the Noether equation

$$
L_{\hat{K}} \hat{\vartheta}=0=d \hat{\vartheta} / d t-\hat{\vartheta} \hat{K}^{\prime, *}-\hat{K}^{\prime} \hat{\vartheta} .
$$

To prove this, it is enough to find by means of the small parameter method, devised before in [24] a non-symmetric $\left(\varphi^{\prime} \neq \varphi^{\prime, *}\right)$ solution $\varphi \in T(\mathcal{M})$ to the following Lie-Lax equation:

$$
d \varphi / d t+\hat{K}^{\prime, *} \varphi=\operatorname{grad} L
$$

for some suitably chosen smooth functional $L \in \mathcal{D}(M)$. As a result of easy calculations one obtains that

$$
\varphi=(-v, 0)^{\top}, \quad L=-\int_{0}^{2 \pi} u v d x .
$$

Making use of (3.11) and the classical Legendrian relationship for the suitable Hamiltonian function

$$
H:=(\varphi, \hat{K})-L,
$$

and the corresponding symplectic structure

$$
\hat{\vartheta}^{-1}:=\varphi^{\prime}-\varphi^{\prime, *}=\left(\begin{array}{cc}
0 & -1 \\
1 & 0
\end{array}\right)
$$

one obtains the implectic structure (3.9) and the corresponding non-singular Hamilton function

$$
\hat{H}_{\vartheta}:=\int_{0}^{2 \pi}\left(v^{2} / 2+v_{x} u^{2}\right) d x .
$$

It is here worth to mention that the determining Lie-Lax equation (3.10) possesses still another solution

$$
\varphi=\left(\frac{u_{x}}{2},-\frac{u_{x}^{2}}{2 v_{x}}\right), \quad L=\frac{1}{4} \int_{0}^{2 \pi} u v_{x} d x,
$$

giving rise, owing to formulas (3.13) and (3.12) to the new co-implectic (singular "symplectic") structure

$$
\hat{\eta}^{-1}:=\varphi^{\prime}-\varphi^{\prime, *}=\left(\begin{array}{cc}
\partial & -\partial u_{x} v_{x}^{-1} \\
-u_{x} v_{x}^{-1} \partial & \frac{1}{2}\left(u_{x}^{2} v_{x}^{-2} \partial+\partial u_{x}^{2} v_{x}^{-2}\right)
\end{array}\right)
$$


and the Hamiltonian functional

$$
\hat{H}_{\eta}:=\frac{1}{2} \int_{0}^{2 \pi}\left(u v_{x}-v u_{x}\right) d x
$$

The co-implectic structure (3.14) is, evidently, singular since $\hat{\eta}^{-1}\left(u_{x}, v_{x}\right)^{\top}=0$. Remark also that, owing to the general symplectic theory results $[1,8,11,15,16,18,24]$ for nonlinear dynamical systems on smooth functional manifolds, operator (3.14) defines on the manifold $\mathcal{M}$ a closed differential two-form. Thereby it is a priori co-symplectic, satisfying on $\mathcal{M}$ the standard Jacobi brackets condition. Moreover, the implectic structure $\hat{\eta}: T^{*}(\mathcal{M}) \rightarrow T^{*}(\mathcal{M})$ satisfies the determining Noether equation

$$
L_{\hat{K}} \hat{\eta}=0=d \hat{\eta} / d t-\hat{\eta} \hat{K}^{\prime, *}-\hat{K}^{\prime} \hat{\eta},
$$

whose solutions can also be obtained by means of the small parameter method, devised before in $[16,24]$. As a result, the second implectic operator has the form

$$
\hat{\eta}:=\left(\begin{array}{cc}
\partial^{-1} & 2 u_{x} \partial^{-1} \\
2 \partial^{-1} u_{x} & 2 v_{x} \partial^{-1}+2 \partial^{-1} v_{x}
\end{array}\right),
$$

giving rise to a new infinite hierarchy of polynomial conservation laws

$$
\hat{\gamma}_{n}:=\int_{0}^{1} d \lambda\left\langle\left(\hat{\vartheta}^{-1} \hat{\eta}\right)^{n} \operatorname{grad} \hat{H}_{\vartheta}[u \lambda\}, u\right\rangle
$$

for all $n \in \mathbb{Z}_{+}$.

In particular, one can easily observe that there hold representations

$$
\frac{d}{d t}(u, v)^{\top}=-\hat{\eta} \operatorname{grad} \hat{H}_{\eta}, \quad \frac{d}{d x}(u, v)^{\top}=-\hat{\vartheta} \operatorname{grad} \hat{H}_{\eta},
$$

where

$$
\hat{H}_{\eta}:=\frac{1}{2} \int_{0}^{2 \pi}\left(u v_{x}-v u_{x}\right) d x
$$

Thereby, one can formulate the following proposition.

Proposition 1. The Riemann type hydrodynamical system (2.1) is a Lax type integrable biHamiltonian flow on the functional manifold $\mathcal{M}$. The corresponding implectic pairs are compatible and given by matrix operators (3.9) and (3.15), the Lax type representation is presented in the differential matrix form (3.6).

Now, making use of (3.16), one can apply the standard reduction procedure upon the corresponding finite dimensional functional subspaces $\mathcal{M}^{2 n} \subset \mathcal{M}, n \in \mathbb{Z}_{+}$, and obtain a large set of exact solutions of special quasi-periodic and solitonic type to dynamical system (2.1) upon the functional submanifold $\mathcal{M}_{\text {red }}$, if the Cauchy data are taken to satisfy constraint (3.8). Here we need to mention that a general solution to the system (2.1), obtained in [23, 27], is presented in an unwieldy involved form, almost completely not feasible for practical applications.

\section{A Riemann type hydrodynamical generalization}

It is here interesting to mention (owing to recent observations by D. Holm for $N=2$ and for arbitrary $N \in \mathbb{Z}_{+}$by M. Pavlov) that the dynamical system (2.1) can be equivalently rewritten up to the time rescaling as

$$
D_{t}^{2} u=0, \quad D_{t}:=\partial / \partial t+u \partial,
$$


under the flow velocity condition $d x / d t:=u$, which is a partial case [5] of the generalized Riemann type hydrodynamic system

$$
D_{t}^{N} u=0
$$

for any integer $N \in \mathbb{Z}_{+}$. If $N=3$, having defined the new variables $v:=D_{t} u, z:=D_{t} v$, one easily obtains the new dynamical system

$$
\left.\begin{array}{l}
u_{t}=v-u u_{x} \\
v_{t}=z-u v_{x} \\
z_{t}=-u z_{x}
\end{array}\right\}:=K[u, v, z]
$$

of hydrodynamical type, which proves also to possess infinite hierarchies of polynomial conservation laws.

As we are interested first in the conservation laws for the system (4.3), the following proposition holds.

Proposition 2. Let $H(\lambda):=\int_{0}^{2 \pi} h(x ; \lambda) d x \in D(\mathcal{M})$ be an almost everywhere smooth functional on the manifold $\mathcal{M}$, depending parametrically on $\lambda \in \mathbb{C}$, and whose density satisfies the differential condition

$$
h_{t}=\lambda(u h)_{x}
$$

for all $t \in \mathbb{R}$ and $\lambda \in \mathbb{C}$ on the solution set of equation (4.1). Then the following iterative differential relationship

$$
(f / h)_{t}=\lambda(u f / h)_{x}
$$

holds, if a smooth function $f \in C^{\infty}(\mathbb{R} ; \mathbb{R})$ (parametrically depending on $\lambda \in \mathbb{C}$ ) satisfies for all $t \in \mathbb{R}$ the linear equation

$$
f_{t}=2 \lambda u_{x} f+\lambda u f_{x}
$$

Proof. We have from (4.4)-(4.6) that

$$
\begin{aligned}
(f / h)_{t} & =f_{t} / h-f h_{t} / h^{2}=f_{t} / h-\lambda f u_{x} / h-\lambda f u h_{x} / h^{2}=f_{t} / h+\lambda f u(1 / h)_{x}-\lambda u_{x} f / h \\
& =\lambda(u f)_{x} / h+\lambda u f(1 / h)_{x}=\lambda(u f / h)_{x},
\end{aligned}
$$

proving the proposition.

The obvious generalization of the previous proposition is read as follows.

Proposition 3. If a smooth function $h \in C^{\infty}(\mathbb{R} ; \mathbb{R})$ satisfies the relationships

$$
h_{t}=k u_{x} h+u h_{x}
$$

where $k \in \mathbb{R}$, then

$$
H=\int_{0}^{2 \pi} h^{1 / k} d x
$$

is a conservation law for the Riemann type hydrodynamical system (2.1). 
The following polynomial dispersionless functionals, constructed by means of Proposition 3, are conserved with respect to the flow (4.3):

$$
\begin{aligned}
H_{n}^{(1)}:=\int_{0}^{2 \pi} d x z^{n}\left(v u_{x}-v_{x} u-\frac{n+2}{n+1} z\right), \\
H^{(4)}:=\int_{0}^{2 \pi} d x\left[-7 v_{x} v^{2} u+z\left(6 z u+2 v_{x} u^{2}-3 v^{2}-4 v u u_{x}\right)\right], \\
H^{(5)}:=\int_{0}^{2 \pi} d x\left(z^{2} u_{x}-2 z v v_{x}\right), \quad H^{(6)}:=\int_{0}^{2 \pi} d x\left(z_{z} v^{3}+3 z^{2} v_{x} u+z^{3}\right), \\
H^{(7)}:=\int_{0}^{2 \pi} d x\left(z_{x} v^{3}+3 z^{2} v u_{x}-3 z^{3}\right), \\
H^{(8)}:=\int_{0}^{2 \pi} d x z\left(6 z^{2} u+3 z v_{x} u^{2}-3 z v^{2}-4 z v u_{x}-2 v_{x} v^{2} u+2 v^{3} u_{x}\right), \\
H^{(9)}:=\int_{0}^{2 \pi} d x\left[1001 v_{x} v^{4} u+\left(1092 z^{2} u^{2}+364 z v_{x} u^{3}-\right.\right. \\
H_{n}^{(2)}:=\int_{0}^{2 \pi} d x z_{x} v z^{n}, \quad H_{n}^{(3)}:=\int_{0}^{2 \pi} d x z_{x}\left(v^{2}-2 z u\right)^{n},
\end{aligned}
$$

where $n \in \mathbb{Z}_{+}$. In particular, as $n=1,2, \ldots$, from (4.3) one obtains that

$$
\begin{aligned}
& H_{0}^{(2)}:=\int_{0}^{2 \pi} d x z_{x} v, \quad H_{1}^{(2)}:=\int_{0}^{2 \pi} d x z_{x} z v, \quad \ldots, \\
& H_{1}^{(3)}:=\int_{0}^{2 \pi} d x z_{x}\left(v^{2}-2 u z\right), \quad H_{2}^{(3)}:=\int_{0}^{2 \pi} d x z_{x}\left(v^{4}+4 z^{2} u^{2}-4 z v^{2} u\right),
\end{aligned}
$$

and so on. Similarly one can construct also infinite hierarchies of conservation laws for the hydrodynamical system (4.3), which are both non-polynomial and dispersive:

$$
\begin{aligned}
& H_{1}^{(1 / 4)}=\int_{0}^{2 \pi} d x\left(-2 u_{x x} u_{x} z_{x}+u_{x x} v_{x}^{2}+2 u_{x}^{2} z_{x x}-u_{x} v_{x x} v_{x}+3 v_{x x} z_{x}-3 v_{x} z_{x x}\right)^{1 / 4}, \\
& H_{2}^{(1 / 3)}=\int_{0}^{2 \pi} d x\left(-v_{x x} z_{x}+v_{x} z_{x x}\right)^{1 / 3} \\
& H_{3}^{(1 / 3)}=\int_{0}^{2 \pi} d x\left(v_{x x} u_{x}-v_{x} u_{x x}-z_{x x}\right)^{1 / 3}, \\
& H_{1}^{(1 / 2)}=\int_{0}^{2 \pi} d x\left[-2 v u_{x} z_{x}+v_{x}^{2}+z\left(-u_{x} v_{x}+3 z_{x}\right)\right]^{1 / 2}, \\
& H_{2}^{(1 / 2)}=\int_{0}^{2 \pi} d x\left(8 u_{x}^{3} z_{x}-3 u_{x}^{2} v_{x}^{2}-18 u_{x} v_{x} z_{x}+6 v_{x}^{3}+9 z_{x}\right)^{1 / 2} \\
& H_{1}^{(1 / 5)}=\int_{0}^{2 \pi} d x\left(-2 u_{x x x} u_{x} z_{x}+u_{x x x} v_{x}^{2}+6 u_{x x}^{2} z_{x}-6 u_{x x} u_{x} z_{x x}\right. \\
& \left.-3 u_{x x} v_{x x} v_{x}+2 u_{x}^{2} z_{x x x}-u_{x} v_{x x x} v_{x}+3 u_{x} v_{x x}^{2}+3 v_{x x x} z_{x}-3 v_{x} z_{x x x}\right)^{1 / 5}, \\
& H_{3}^{(1 / 3)}=\int_{0}^{2 \pi} d x\left[k_{1} u\left(-v_{x x} z_{x}+v_{x} z_{x x}\right)+k_{1} v\left(u_{x x} z_{x}-u_{x} z_{x x}\right)\right. \\
& \left.+z\left(k_{2} u_{x x} v_{x}-k_{2} u_{x} v_{x x}+k_{1} z_{x x}+k_{2} z_{x x}\right)+k_{3}\left(-3 u_{x} v_{x} z_{x}+v_{x}^{3}+3 z_{x}^{2}\right)\right]^{1 / 3}, \quad \ldots,
\end{aligned}
$$

and so on, where $k_{j} \in \mathbb{R}, j=1,2,3$, are arbitrary real numbers. The problem which remains still open consists in proving, if any, that the generalized hydrodynamical system (4.3) is a Lax type 
integrable bi-Hamiltonian flow on the periodic functional manifold $\mathcal{M}:=C^{(\infty)}\left(\mathbb{R} / 2 \pi \mathbb{Z} ; \mathbb{R}^{3}\right)$, as it was proved above for the system (4.2) at $N=2$. This problem will be analyzed in the Section below.

\section{The Hamiltonian analysis}

Consider the system (4.3) as a nonlinear dynamical system

$$
\left.\begin{array}{l}
u_{t}=v-u u_{x} \\
v_{t}=z-u v_{x} \\
z_{t}=-u z_{x}
\end{array}\right\}:=K[u, v, z],
$$

on the $2 \pi$-periodic smooth functional manifold $\mathcal{M}$ and analyze it from the Hamiltonian point of view. To tackle with this problem, it is enough to construct $[7,11,24]$ exact non-symmetric solutions to the Lie-Lax equation

$$
d \varphi / d t+K^{\prime, *} \varphi=\operatorname{grad} L, \quad \varphi^{\prime} \neq \varphi^{\prime, *},
$$

for some functional $L \in D(\mathcal{M})$, where $\varphi \in T^{*}(\mathcal{M})$ is, in general, a quasi-local vector, such that the system (4.3) allows the following Hamiltonian representation:

$$
K[u, v, z]=-\eta \operatorname{grad} H[u, v, z], \quad H=(\varphi, K)-L, \quad \eta^{-1}=\varphi^{\prime}-\varphi^{\prime, *} .
$$

As a test solution to (5.2) one can take the one

$$
\varphi=\left(u_{x} / 2,0,-z_{x}^{-1} u_{x}^{2} / 2+z_{x}^{-1} v_{x}\right)^{\top}, \quad L=\frac{1}{2} \int_{0}^{2 \pi}\left(2 z+v u_{x}\right) d x,
$$

which gives rise to the following co-implectic operator:

$$
\eta^{-1}:=\varphi^{\prime}-\varphi^{\prime, *}=\left(\begin{array}{ccc}
\partial & 0 & -\partial u_{x} z_{x}^{-1} \\
0 & 0 & \partial z_{x} \\
-u_{x} z_{x}^{-1} \partial & z_{x} \partial & \frac{1}{2}\left(u_{x}^{2} z_{x}^{-2} \partial+\partial u_{x}^{2} z_{x}^{-2}\right) \\
& & -\left(v_{x} z_{x}^{-2} \partial+\partial v_{x} z_{x}^{-2}\right)
\end{array}\right) .
$$

This expression is not strictly invertible, as its kernel possesses the translation vector field $d / d x: \mathcal{M} \rightarrow T(\mathcal{M})$ with components $\left(u_{x}, v_{x}, z_{x}\right)^{\top} \in T(\mathcal{M})$, that is $\eta^{-1}\left(u_{x}, v_{x}, z_{x}\right)^{\top}=0$.

Nonetheless, upon formal inverting the operator expression (5.3), we obtain by means of simple enough, but slightly cumbersome, direct calculations, that the Hamiltonian function equals

$$
H:=\int_{0}^{2 \pi} d x\left(u_{x} v-z\right) .
$$

and the implectic $\eta$-operator looks as

$$
\eta:=\left(\begin{array}{ccc}
\partial^{-1} & u_{x} \partial^{-1} & 0 \\
\partial^{-1} u_{x} & v_{x} \partial^{-1}+\partial^{-1} v_{x} & \partial^{-1} z_{x} \\
0 & z_{x} \partial^{-1} & 0
\end{array}\right) .
$$

The same way, representing the Hamiltonian function (5.4) in the scalar form

$$
H=\left(\psi,\left(u_{x}, v_{x}, z_{x}\right)^{\top}\right), \quad \psi=\frac{1}{2}\left(-v, u+\cdots,-\frac{1}{\sqrt{z}} \partial^{-1} \sqrt{z}\right)^{\top},
$$


one can construct a second implectic (co-symplectic) operator $\vartheta: T^{*}(\mathcal{M}) \rightarrow T(\mathcal{M})$, looking up to $O\left(\mu^{2}\right)$ terms, as follows:

$$
\vartheta=\left(\begin{array}{ccc}
\mu\left(\frac{\left(u^{(1)}\right)^{2}}{z^{(1)}} \partial+\partial \frac{\left(u^{(1)}\right)^{2}}{z^{(1)}}\right) & 1+\frac{2 \mu}{3}\left(\frac{u^{(1)} v^{(1)}}{z^{(1)}} \partial\right. & \frac{2 \mu}{3}\left(\partial \frac{\left(v^{(1)}\right)^{2}}{z^{(1)}}+\partial u^{(1)}\right) \\
-1+\frac{2 \mu}{3}\left(\partial \frac{u^{(1)} v^{(1)}}{z^{(1)}}\right. & \frac{2 \mu}{3}\left(\frac{\left(v^{(1)}\right)^{2}}{z^{(1)}} \partial+\partial \frac{\left(v^{(1)}\right)^{2}}{z^{(1)}}\right) & \\
\left.+2 \frac{u^{(1)} v^{(1)}}{z^{(1)}} \partial\right) & +\frac{2 \mu}{3}\left(u^{(1)} \partial+\partial u^{(1)}\right) & 2 \mu \partial v^{(1)} \\
\frac{2 \mu}{3}\left(\frac{\left(v^{(1)}\right)^{2}}{z^{(1)}} \partial+u^{(1)} \partial\right) & 2 \mu v^{(1)} \partial & \mu\left(\partial z^{(1)}+z^{(1)} \partial\right)
\end{array}\right)+O\left(\mu^{2}\right)
$$

where we put, by definition, $\vartheta^{-1}:=\left(\psi^{\prime}-\psi^{\prime, *}\right), u:=\mu u^{(1)}, v:=\mu v^{(1)}, z:=\mu z^{(1)}$ as $\mu \rightarrow 0$, and whose exact form needs some additional simple enough but cumbersome calculations, which will be presented in a work under preparation.

The operator (5.7) satisfies the Hamiltonian vector field condition:

$$
\left(u_{x}, v_{x}, z_{x}\right)^{\top}=-\vartheta \operatorname{grad} H
$$

following easily from (5.6).

Now having applied to the pair of implectic operators the gradient-holonomic scheme [11, 16, 24] of constructing a Lax type representation for the dynamical system (5.1) we obtain by means of slightly cumbersome and tedious calculations the following compatible Lax type representation:

$$
\begin{aligned}
& f_{x}=\ell[u, v ; \lambda] f, \quad f_{t}=p(\ell) f, \quad p(\ell):=-u \ell[u, v ; \lambda]+q(\lambda), \\
& \ell\left[u, v, z ; \lambda=\left(\begin{array}{ccc}
\lambda u_{x} & -v_{x} & z_{x} \\
3 \lambda^{2} & -2 \lambda u_{x} & \lambda v_{x} \\
\lambda^{2} r[u, v, z] & -3 \lambda & \lambda u_{x}
\end{array}\right), \quad q(\lambda):=\left(\begin{array}{ccc}
0 & 0 & 0 \\
\lambda & 0 & 0 \\
0 & 1 & 0
\end{array}\right),\right. \\
& p(\ell)=\left(\begin{array}{ccc}
-\lambda u u_{x} & u v_{x} & -u z_{x} \\
-3 u \lambda^{2}+\lambda & 2 \lambda u u_{x} & -\lambda u v_{x} \\
-\lambda^{2} r[u, v, z] u & 1+3 u \lambda & -\lambda u u_{x}
\end{array}\right),
\end{aligned}
$$

where $f \in C^{\infty}\left(\mathbb{R} ; \mathbb{C}^{3}\right), \lambda \in \mathbb{C} \backslash\{0\}$ is a spectral parameter and $r: \mathcal{M} \rightarrow \mathbb{R}$ is a smooth mapping, satisfying the differential equation

$$
D_{t} r+u_{x} r=6
$$

The latter possesses a wide set $\mathcal{R}$ of different solutions, amongst which there are the following:

$$
\begin{aligned}
r \in \mathcal{R}:= & \left\{\left(6 x v-3 u^{2}\right) / z\right]_{x}, 3\left(2 v_{x}-u_{x}^{2}\right) z_{x}^{-1}, \frac{2 u_{x}^{3}-6 u_{x} v_{x}+9 z_{x}}{2 u_{x} z_{x}-v_{x}^{2}}, \\
& \left.\left(v_{x} v^{3}-3 u_{x} v^{2} z+u z_{x}\left(u z-v^{2}\right)+6 v z^{2}\right) z^{-3}\right\} .
\end{aligned}
$$

Thereby, the following proposition holds.

Proposition 4. The generalized Riemann type hydrodynamical equation (4.2) at $N=2$ and $N=3$ is equivalent to Lax type integrable bi-Hamiltonian dynamical systems (2.1) and (5.1), whose Hamiltonian structures and Lax type representations are given by expressions (3.13), (3.15), (3.6), and (5.5), (5.7), (5.8), (5.9), respectively. 
Note here that only the third element from the set (5.9) allows the reduction $z=0$ to the case $N=2$. Concerning the case $N=4$ and the general case $N \in \mathbb{Z}_{+}$, applying successively the method devised above, one can obtain [3] for the Riemann type hydrodynamical system (5.1) both infinite hierarchies of dispersive and dispersionless conservation laws, their symplectic structures and the related Lax type representations, which is a topic of the next work under preparation.

\section{Acknowledgments}

M.P. and A.P. are appreciated to Organizers of the Symmetry-2009 Conference (June 21-27, 2009) held in Kyiv, Ukraine, and the NEEDS-2009 Conference (May 15-23, 2009), held in Isola Rossa of Sardinia, Italy, for the invitations to deliver reports and for a partial support. M.P. was, in part, supported by RFBR grant 08-01-00054 and a grant of the RAS Presidium "Fundamental Problems of Nonlinear Dynamics". The authors thanks go to Professors M. Błaszak, N. Bogolubov (jr.) and D. Blackmore for useful discussions of the results obtained. Authors are also cordially thankful to Referees who have read the article and made very important remarks and suggestions, which were very instrumental for final preparing a manuscript, and which made it possible both to improve and correct the exposition.

\section{References}

[1] Błaszak M., Multi-Hamiltonian theory of dynamical systems, Texts and Monographs in Physics, SpringerVerlag, Berlin, 1998.

[2] Bogolyubov N.N. Jr., Prykarpatsky A.K., Gucwa I., Golenia J., Analytical properties of an OstrovskyWhitham type dynamical system for a relaxing medium with spatial memory and its integrable regularization, Preprint IC/2007/109, Trieste, Italy, 2007, arXiv:0902.4395.

[3] Bogolyubov N.N. Jr., Golenia J., Popowicz Z., Pavlov M.V., Prykarpatsky A.K., A new Riemann type hydrodynamical hierarchy and its integrability analysis, Preprint IC/2009/095, Trieste, Italy, 2009.

[4] Brunelli J.C., Das A., On an integrable hierarchy derived from the isentropic gas dynamics, J. Math. Phys. 45 (2004), 2633-2645, nlin.SI/0401009.

[5] Chorin A.J., Marsden J.E., A mathematical introduction to fluid mechanics, 3rd ed., Texts in Applied Mathematics, Vol. 4, Springer-Verlag, New York, 1993.

[6] Davidson R.C., Methods in nonlinear plasma theory, Academic Press, New York, 1972.

[7] Faddeev L.D., Takhtajian L.A., Hamiltonian methods in the theory of solitons, Classics in Mathematics, Springer, Berlin, 2007.

[8] Fuchssteiner B., Fokas A.S., Symplectic structures, their Bäcklund transformations and hereditary symmetries, Phys. D 4 (1981), 47-66.

[9] Gurevich A.V., Zybin K.P., Nondissipative gravitational turbulence, Soviet Phys. JETP 67 (1988), 1-12.

[10] Gurevich A.V., Zybin K.P., Large-scale structure of the Universe. Analytic theory, Soviet Phys. Usp. 38 (1995), 687-722.

[11] Hentosh O., Prytula M., Prykarpatsky A., Differential-geometric and Lie-algebraic foundations of investigating nonlinear dynamical systems on functional manifolds, 2nd ed., Lviv University Publ., 2006 (in Ukrainian).

[12] Hunter J.K., Saxton R., Dynamics of director fields, SIAM J. Appl. Math. 51 (1991), 1498-1521.

[13] Hunter J.K., Zheng Y.X., On a completely integrable nonlinear hyperbolic variational equation, Phys. D 79 (1994), 361-386.

[14] Lenells J., The Hunter-Saxton equation: a geometric approach, SIAM J. Math. Anal. 40 (2008), $266-277$.

[15] Magri F., A simple model of the integrable Hamiltonian equations, J. Math. Phys. 19 (1978), 1156-1162.

[16] Mitropol'skij Yu.A., Bogolyubov N.N. Jr., Prikarpatskij A.K., Samojlenko V.G., Integrable dynamical systems: spectral and differential-geometric aspects, Naukova Dumka, Kiev, 1987 (in Russian). 
[17] Morrison A.J., Parkes E.J., Vakhnenko V.O., The $N$ loop soliton solution of the Vakhnenko equation, Nonlinearity 12 (1999), 1427-1437.

[18] Olver P.J., Applications of Lie groups to differential equations, Graduate Texts in Mathematics, Vol. 107, Springer-Verlag, New York, 1986.

[19] Olver P.J., Rosenau P., Tri-Hamiltonian duality between solitons and solitary-wave solutions having compact support, Phys. Rev. E 53 (1996), 1900-1906.

[20] Ostrovsky L.A., Nonlinear internal waves in a rotating ocean, Oceanology 18 (1978), 119-125.

[21] Parkes E.J., The stability of solution of Vakhnenko's equation, J. Phys. A: Math. Gen. 26 (1993), 6469-6475.

[22] Parkes E.J., Vakhnenko V.O., Explicit solutions of the Camassa-Holm equation, Chaos Solitons Fractals 26 (2005), 1309-1316.

[23] Pavlov M.V., The Gurevich-Zybin system, J. Phys. A: Math. Gen. 38 (2005), 3823-3840, nlin.SI/0412072.

[24] Prykarpatsky A.K., Mykytyuk I.V., Algebraic integrability of nonlinear dynamical systems on manifolds. Classical and quantum aspects, Mathematics and its Applications, Vol. 443, Kluwer Academic Publishers Group, Dordrecht, 1998.

[25] Prykarpatsky A.K., Prytula M.M., The gradient-holonomic integrability analysis of a Whitham-type nonlinear dynamical model for a relaxing medium with spatial memory, Nonlinearity 19 (2006), 2115-2122.

[26] Prykarpatsky A.K., Prytula M.M., The gradient-holonomic analysis of the integrability of a nonlinear Whitham-type model for a relaxing medium with memory, Dopov. Nats. Akad. Nauk Ukr. Mat. Prirodozn. Tekh. Nauki (2006), no. 5, 13-18 (in Ukrainian).

[27] Sakovich S., On a Whitham-type equation, SIGMA 5 (2009), 101, 7 pages, arXiv:0909.4455.

[28] Whitham G.B., Linear and nonlinear waves, Wiley-Interscience, New York - London - Sydney, 1974. 